\title{
PERSPECTIVAS DE DOCENTES DE ENFERMAGEM DOS DOMÍNIOS DO CORE COMPETENCIES FRAMEWORK FOR HEALTH PROMOTION
}

\author{
Ítala Keane Rodrigues Dias ${ }^{1}$ Olga Feitosa Braga Teixeira², Ilara Parente Pinheiro Teodoro ${ }^{3}$, \\ Evanira Rodrigues Maia ${ }^{4}$, Maria do Socorro Vieira Lopes ${ }^{4}$, \\ Maria de Fátima Antero Sousa Machado ${ }^{5}$
}

\begin{abstract}
RESUMO: Objetivo: verificar as perspectivas de docentes de um curso de graduação em enfermagem acerca dos domínios do Core Competencies Framework for Health Promotion, e como estes estão presentes em sua prática. Método: o estudo foi realizado no ano de 2017, em uma instituição pública de ensino superior da Região Nordeste. Foram convidados 62 docentes que atenderam aos critérios de inclusão e exclusão estabelecidos, dos quais 15 participaram da pesquisa. Os resultados foram analisados a partir da análise de conteúdo. Resultados: emergiram as categorias: "Saberes dos docentes acerca da promoção da saúde: reconhecendo os domínios" e "Práticas dos docentes em promoção da saúde: aplicabilidade dos domínios do Core Competencies Framework for Health Promotion". Divergências encontradas a respeito dos saberes e práticas podem influenciar a formação dos enfermeiros em relação às competências necessárias à promoção da saúde. Conclusão: é importante oportunizar estratégias de qualificação e formação continuada em torno da temática em questão.
\end{abstract}

DESCRITORES: Competência profissional; Promoção da saúde; Educação em enfermagem; Docentes de enfermagem; Atenção à saúde.

\section{NURSING EDUCATORS' PERCEPTIONS OF THE DOMAINS OF THE CORE COMPETENCIES FRAMEWORK FOR HEALTH PROMOTION}

ABSTRACT: Objective: to investigate the perceptions of nursing professors from an undergraduate course of the domains of the Core Competencies Framework for Health Promotion and find out how these domains are applied in their teaching practice. Method: The study was conducted in 2017 at a public institution of higher education in the Northeastern Region of Brazil. Sixty-two (62) teachers who met the established inclusion and exclusion criteria were invited. Of these, 15 participated in the study. Results: The following categories emerged: "Nursing educators" knowledge about health promotion: recognizing the domains" and "Nurse Educators" Practices in Health Promotion: Application of the domains of the Core Competencies Framework for Health Promotion." Conclusion: Training and continuing education strategies on the referred subject should be provided.

KEYWORDS: Professional competence; Health promotion; Nursing education; Nursing teachers; Healthcare.

\section{PERSPECTIVAS DE LOS DOMINIOS DEL CORE COMPETENCIES FRAMEWORK FOR HEALTH PROMOTION POR DOCENTES DE ENFERMERÍA}

RESUMEN: Objetivo: verificar las perspectivas de docentes de un curso de graduación en enfermería acerca de los dominios del Core Competencies Framework for Health Promotion, y como esos dominios se encuentran en su práctica. Método: el estudio ocurrió en el año de 2017, en una institución pública de enseñanza superior de la Región Nordeste. Se invitaron 62 docentes que obedecían a los criterios de inclusión y exclusión establecidos, de los cuales 15 participaron de la investigación. Se analizaron los resultados por medio del análisis de contenido. Resultados: las categorías resultantes fueron: "Saberes de los docentes acerca de la promoción de la salud: reconociendo los dominios" y "Prácticas de los docentes en promoción de la salud: aplicabilidad de los dominios del Core Competencies Framework for Health Promotion". Hubo divergencias acerca de los saberes y prácticas que pueden influenciar la formación de los enfermeros sobre las competencias necesarias a la promoción de la salud. Conclusión: es importante promover estrategias de cualificación y formación continuada al rededor de la temática en cuestión.

DESCRIPTORES: Competencia profesional; Promoción de la salud; Educación en enfermería; Docentes de enfermería; Atención a la salud.

${ }^{1}$ Enfermeira. Mestranda do programa de pós-graduação em Enfermagem da Universidade Regional do Cariri. Enfermeira do Instituto Federal do Ceará. Tauá, CE, Brasil.

${ }^{2}$ Enfermeira. Mestranda do programa de pós-graduação em Enfermagem da Universidade Regional do Cariri. Docente da Escola Técnica de Saúde de Cajazeiras da Universidade Federal de Campina Grande. Cajazeira, PB, Brasil.

${ }^{3}$ Enfermeira. Mestranda do programa de pós-graduação em Enfermagem da Universidade Regional do Cariri. Crato, Ceará, Brasil.

${ }^{4}$ Enfermeira. Doutora em Enfermagem pela Universidade Federal do Ceará. Docente do curso de Enfermagem e do programa de pós-graduação em Enfermagem da Universidade Regional do Cariri. Crato, CE, Brasil.

${ }^{5}$ Enfermeira. Doutora em Enfermagem pela Universidade Federal do Ceará. Docente do programa de pós-graduação em Enfermagem da Universidade Regional do Cariri. Crato, CE, Brasil. 


\section{INTRODUÇÃO}

Competência é uma palavra do senso comum utilizada para designar uma pessoa qualificada para realizar alguma tarefa. A competência de um indivíduo não é um estado e não deve ser reduzida a um conhecimento específico; pelo contrário, é formada por um conjunto de aprendizagens sociais nutrido pela formação educacional e pela experiência profissional, o que implica saber mobilizar, articular, integrar e transferir os conhecimentos, recursos e habilidades, segundo o contexto profissional ${ }^{(1)}$.

Existem muitas definições para competência. Todas elas se referem ao perfil profissional exigido no local de trabalho e às exigências de saber necessárias para um desempenho adequado ${ }^{(2)}$. No campo específico da promoção da saúde (PS), competência é definida como uma combinação de conhecimentos, habilidades e atitudes, a qual possibilita que um indivíduo desempenhe tarefas de acordo com um padrão ${ }^{(3)}$.

As competências de PS do Core Competencies Framework for Health Promotion (CompHP) têm como objetivo principal a formação de um consenso a partir do qual se estabeleçam métodos para a implementação de padrões, visando à inovação e a melhores práticas em saúde ${ }^{(4)}$.

As competências para PS do CompHP podem ser usadas na formação de promotores da saúde, quer seja inicialmente, quer no desenvolvimento profissional contínuo durante a carreira. Elas servem, então, como critérios para avaliação de qualificação em PS ou de experiências que venham da prática(5).

No CompHP, o conjunto de 47 competências necessárias para desenvolver ações eficazes em PS está dividido em nove domínios e abrange valores (equidade, justiça social, ética, autonomia dos indivíduos), habilidades (princípios conceituais da PS) e conhecimentos. Cada domínio especifica os conhecimentos, as habilidades e os critérios de desempenho exigidos para demonstrar a aquisição das competências. Os nove domínios contidos no CompHP são: (1) favorecimento de mudanças, (2) advocacia em saúde, (3) parceria, (4) comunicação, (5) liderança, (6) diagnóstico, (7) planejamento, (8) implementação e (9) avaliação e pesquisa ${ }^{(4)}$.

A procura por capacitação e formação profissional na área de PS é uma demanda crescente, porém ainda embrionária no Brasil. Instituições de Ensino Superior renomadas têm organizado cursos de especialização, mestrado e doutorado nessa área, o que denota crescente envolvimento com tal formação como campo de teorias e práticas ${ }^{(4)}$.

No Brasil, estudos que tratam da formação profissional para a PS também são exíguos. No âmbito da graduação, o ensino da PS é igualmente incipiente. Embora existam estudos que defendam a inserção dessa formação nos cursos de graduação, uma das dificuldades apontadas para sua introdução relacionase à imprecisão conceitual que permeia tal campo e como esta não exatidão manifesta-se na formação e na atenção desenvolvida pelos profissionais no cotidiano dos serviços de saúde brasileiros ${ }^{(6)}$.

O objetivo deste estudo foi verificar as perspectivas de docentes de um curso de graduação em enfermagem acerca dos domínios do CompHP, e como estes estão presentes em sua prática.

\section{MÉTODO}

Trata-se de um estudo com abordagem qualitativa, realizado no ano de 2017, em uma Instituição de Ensino Superior pública da Região Nordeste. Na Região Metropolitana onde o estudo foi desenvolvido, existiam quatro Instituições de Ensino Superior que disponibilizavam graduação em enfermagem. $\mathrm{O}$ local foi escolhido considerando a facilidade de acesso dos pesquisadores.

O critério de inclusão dos participantes foi ser docente do curso de graduação em enfermagem do campus escolhido para o estudo. Foram excluídos docentes que não possuíssem graduação em enfermagem. 
A coleta de dados foi realizada mediante aplicação de questionário on-line enviado para o e-mail dos 62 docentes que atenderam aos critérios de inclusão. O questionário permaneceu disponível durante 30 dias em um diretório eletrônico. Foram devolvidos 15 questionários, que constituíram a amostra deste estudo.

Os dados foram analisados de acordo com a Análise de Conteúdo Temática. Os depoimentos dos participantes foram denominados de D1 a D15 para garantir anonimato.

O questionário abordava os perfis etário, social, acadêmico e profissional. As perguntas sobre o CompHP foram submetidas a um pré-teste, do qual participaram dois docentes do curso de graduação em enfermagem não envolvidos na pesquisa. Depois da aplicação do pré-teste, alguns itens foram acrescentados, e outros, modificados.

O projeto de pesquisa foi aprovado pelo Comitê de Ética e Pesquisa com o parecer $n . \stackrel{0}{2} .005 .435$. O Termo de Consentimento Livre e Esclarecido (TCLE) foi enviado aos participantes juntamente com o questionário.

\section{RESULTADOS}

Participaram do estudo 15 docentes, com idade entre 25 e 65 anos, sendo 11 mulheres. Todos eram especialistas em diferentes áreas da saúde (Gerontologia, Obstetrícia, Saúde da Família, Docência no Ensino Superior, Saúde da Criança, Bioquímica e Biologia Molecular, Administração Hospitalar e Gestão em Saúde); 11 tinham mestrado, e três, doutorado.

Todos os participantes tinham vínculo formal com a instituição. Apenas um referiu ter também outro vínculo empregatício. O tempo mínimo de atuação docente foi de um ano (quatro docentes) e o máximo, 17 anos (um docente), com média de 6 anos e 5 meses.

Foram identificadas duas categorias: "Saberes dos docentes acerca da promoção da saúde: reconhecendo os domínios do CompHP" e "Práticas dos docentes em promoção da saúde: aplicabilidade dos domínios do CompHP".

\section{Categoria 1: Saberes dos docentes acerca da promoção da saúde: reconhecendo os domínios do CompHP}

O Quadro 1 evidencia alguns domínios identificados no discurso dos participantes, configurados em seus saberes.

Quadro 1 - Saberes dos docentes acerca da promoção da saúde evidenciados por meio do conhecimento dos domínios do Core Competencies Framework for Health Promotion (CompHP). Crato, CE, Brasil, 2016. (continua)

\begin{tabular}{|c|c|}
\hline Domínio & Evidência de conhecimento do CompHP \\
\hline \multirow{2}{*}{$\begin{array}{l}\text { Possibilidade } \\
\text { de mudanças }\end{array}$} & $\begin{array}{l}\text { É trabalhar capacitando pessoas e comunidades para que elas próprias (empoderamento) sejam } \\
\text { capazes de modificarem fatores negativos que afetem suas vidas (por exemplo: saneamento, água, } \\
\text { educação)... possam melhorar sua qualidade de vida. (D6) }\end{array}$ \\
\hline & $\begin{array}{l}\text { Entendo promoção da saúde como a capacitação dos indivíduos e coletividades para atuarem em } \\
\text { benefício de sua saúde e qualidade de vida. (D10) }\end{array}$ \\
\hline \multirow[b]{2}{*}{ Parceria } & $\begin{array}{l}\text { Estratégia transversal, integrada e intersetorial para promoção da qualidade de vida e saúde das } \\
\text { pessoas, visando reduzir as vulnerabilidades, prevenir doenças e agravos, contudo em um ambien- } \\
\text { te preservado e saudável. (D2) }\end{array}$ \\
\hline & $\begin{array}{l}\text { Promover saúde pode traduzir-se na construção da melhoria da qualidade de vida e saúde de } \\
\text { uma pessoa ou de uma comunidade, por uma equipe interdisciplinar, a partir dos conhecimentos } \\
\text { prévios dessa comunidade e da sua participação nesse processo, com ênfase no pensamento so- } \\
\text { cioambiental. (D15) }\end{array}$ \\
\hline
\end{tabular}




\begin{tabular}{|c|c|}
\hline Comunicação & Consiste na oferta de informações educativas que evitem o processo de adoecimento. (D13) \\
\hline Liderança & $\begin{array}{l}\text { Trata-se de estabelecer subsídios para que o ser cuidado atue de maneira corresponsável no pro- } \\
\text { cesso saúde-doença, além de transformá-lo em um agente multiplicador de ações que valorizem a } \\
\text { saúde em todos os seus aspectos. (D7) }\end{array}$ \\
\hline \multirow[t]{2}{*}{ Diagnóstico } & $\begin{array}{l}\text { Promoção da saúde é um processo que envolve aspectos que devem ser potencializadores dos } \\
\text { determinantes da saúde. Como processo, à medida que se promove a saúde, previnem-se agravos. } \\
\text { Portanto, aspectos sociais, culturais, históricos, políticos, ambientais e pessoais devem ser incluí- } \\
\text { dos no âmbito do promover saúde. (D4) }\end{array}$ \\
\hline & $\begin{array}{l}\text { Pela epidemiologia, poderíamos situar a promoção da saúde como ações amplas e múltiplas de- } \\
\text { senvolvidas no contexto de vida das pessoas em seus contextos e ambientes, que visam potencia- } \\
\text { lizar determinantes de saúde em suas diferentes dimensões. (D12) }\end{array}$ \\
\hline
\end{tabular}

Dos nove domínios, somente cinco foram evidenciados nas falas dos docentes. Além disso, os saberes desses domínios foram mencionados por participantes diferentes, ou seja, nenhum docente demonstrou ter propriedade em mais de um domínio do CompHP.

\section{Categoria 2: Práticas dos docentes em promoção da saúde: aplicabilidade dos domínios do CompHP}

O Quadro 2 apresenta a aplicabilidade dos domínios do CompHp na prática docente, de acordo com as respostas dos participantes.

Quadro 2 - Domínios do Core Competencies Framework for Health Promotion (CompHP) presentes na prática docente, de acordo com as respostas dos participantes. Crato, CE, Brasil, 2016

\begin{tabular}{|c|c|}
\hline Domínio & Evidência de presença na prática \\
\hline $\begin{array}{l}\text { Favorecimento de } \\
\text { mudanças }\end{array}$ & $\begin{array}{l}\text { Na disciplina saúde da mulher, tentamos o empoderamento das mesmas no intuito de } \\
\text { produzir mudanças e melhoria de sua saúde. (D3) }\end{array}$ \\
\hline Advocacia em saúde & $\begin{array}{l}\text { Instigar no aluno a consciência crítica relacionada ao pensamento de que a melhoria da } \\
\text { qualidade de vida das pessoas está relacionada à promoção da saúde ambiental. (D15) }\end{array}$ \\
\hline Parceria & Mediar através de parceria [...] (D9) \\
\hline Comunicação & $\begin{array}{l}\text { Estabeleço duas ferramentas que considero importante: a informação (subsídio para } \\
\text { comunicação) e a sensibilização (pressuposto para educação consciente e transformadora) } \\
\text { sobre o contexto vivenciado no Sistema Único de Saúde. (D12) } \\
\text { Treinamento de habilidades cognitivas e instrumentais - prática dialógica e dialética. (D4) }\end{array}$ \\
\hline Liderança & $\begin{array}{l}\text { [...] desenvolver ações preventivas, promovendo a autonomia das pessoas, visando melhorar } \\
\text { sua qualidade de vida. (D15) }\end{array}$ \\
\hline Diagnóstico & $\begin{array}{l}\text { [...] identificar fatores de risco para a saúde; identificar situações de fragilidade [...] (D15) } \\
\text { [...] desenvolvemos as habilidades embasadas no conhecimento científico. (D3) }\end{array}$ \\
\hline Planejamento & $\begin{array}{l}\text { Gosto de propor aos alunos que realizem atividades educativas e que estas sejam planejadas } \\
\text { de acordo com as necessidades dos usuários. Assim possibilita que as atividades tenham } \\
\text { mais êxito. (D2) }\end{array}$ \\
\hline Implementação & $\begin{array}{l}\text { Preparar o aluno para intervir positivamente no processo saúde-doença, de forma a respeitar } \\
\text { o princípio da integralidade, observando as pessoas na sua totalidade, em seu contexto. } \\
\text { (D15) }\end{array}$ \\
\hline & Promoção do debate e reflexão crítica sobre os aspectos da sociedade moderna. (D4) \\
\hline Avaliação e pesquisa & $\begin{array}{l}\text { [...] realização de pesquisas na área de promoção da saúde. (D14) } \\
\text { [...] humanização e prática baseada em evidência [...]. (D10) }\end{array}$ \\
\hline
\end{tabular}

Todos os domínios do CompHP puderam ser evidenciados na prática docente, de acordo com as respostas dos participantes. Ainda, nesta categoria alguns docentes (D3, D4 e D15) fizeram menção do emprego de mais de um dos domínios em suas práticas docentes. 


\section{DISCUSSÃO}

O domínio favorecimento de mudanças é descrito no CompHP como a capacidade de indivíduos, grupos, comunidades e organizações para construir ações de PS, a fim de melhorar a saúde e reduzir as desigualdades neste âmbito ${ }^{(5)}$. Esse domínio esteve presente tanto nos saberes dos participantes (Quadro 1) como em sua prática (Quadro 2), na perspectiva de PS. Considerar esse aspecto na formação de enfermeiros permite produzir conhecimentos e fomentar práticas sociais hegemônicas e dominantes no campo da saúde, proporcionando mudança no processo de formação dos alunos.

O domínio advocacia em saúde, identificado apenas na prática docente (Quadro 2), reafirma os achados da literatura. Embora existam documentos norteadores da PS que reconheçam a advocacia em saúde como prioridade, esse domínio ainda é pouco explorado. Entre os fatores que contribuem para tal estão falta de tempo, outras prioridades, frustração com o processo, escassez de recursos e dúvidas sobre o impacto de ações individuais ${ }^{(7)}$.

Deste modo, torna-se indispensável que os docentes enfatizem, em suas abordagens de PS, as legislações que asseguram à população o direito à saúde como um dever do Estado, para que os graduandos se apropriem dos direitos dos usuários do Sistema Único de Saúde (SUS) e sejam capazes de persuadir e argumentar a favor da população. Instigado a ter consciência crítica, o aluno passa a ter a capacidade de formular suas opiniões em relação à saúde, argumentar e reivindicar a favor dos usuários.

O domínio parceria revela o valor do envolvimento de setores/disciplinas para a promoção da qualidade de vida e saúde, a partir das necessidades da comunidade. Os achados desta pesquisa demonstram que, com relação à intersetorialidade, os docentes têm preocupações de compartilhar tecnologias e de desfrutar diretamente os benefícios, além de propiciar a formulação de um saber crítico-reflexivo.

A PS, em uma visão interdisciplinar, deve vislumbrar a melhoria das condições de vida da população e o reconhecimento do direito de cidadania, tendo como princípios a concepção holística da saúde, a equidade, a intersetorialidade, a participação social e a sustentabilidade ${ }^{(8)}$.

Em instituições de ensino, esse domínio pode ser potencializado, ao envolver o aluno em projetos de extensão, pesquisa de Iniciação Científica, grupos de pesquisas, entre outros, proporcionando a capacidade de atuar concomitantemente em diferentes serviços e setores, o que certamente fortalece as ações de $\mathrm{PS}^{(9)}$.

A parceria, presente nos saberes e práticas dos docentes investigados, pode ser desenvolvida no ensino de diversas maneiras, buscando contribuir para a integração ensino/serviço/comunidade/usuário. Podemos citar, por exemplo, as parcerias entre os serviços de saúde existentes na localidade; entre os diferentes componentes curriculares do curso e de outras áreas do conhecimento; com outras instituições de ensino etc. Os graduandos devem ser estimulados a ultrapassar as barreiras extramuros da universidade, complementando seu processo formativo e percebendo os impactos das parcerias, para tornar as ações planejadas efetivas e eficazes.

A comunicação representa um dos domínios que exerce grande aplicabilidade na docência. O professor dispõe de uma diversidade de técnica e tecnologias, que facilita o processo de ensino e aprendizagem. Inclusive, alguns participantes deste estudo sinalizaram esse domínio por intermédio da prática dialógica, dialética e de disseminação de informações.

Nos achados do estudo houve a preocupação de trabalhar com estratégias mediante informações educativas, para evitar o adoecimento. Esse domínio exerce a transmissão de ações de PS de forma efetiva, utilizando técnicas e tecnologia de informações apropriadas para públicos diversos ${ }^{(5)}$.

A comunicação tem sido um dos instrumentos de trabalho do enfermeiro. Estes profissionais, em seu processo formativo, precisam de subsídios que estimulem a aquisição desse domínio, de forma integrada e significativa, empregando os diversos recursos tecnológicos e midiáticos, que podem proporcionar uma comunicação que atenda às necessidades do público assistido ${ }^{(10)}$. 
A presença desse domínio em ambas as categorias (Quadros 1 e 2) revela a importância em capacitar o indivíduo, que deve tornar-se autônomo no processo saúde-doença, sendo estimulado a compartilhar ações em PS.

A liderança - presente nos saberes e práticas dos docentes acerca da promoção da saúde nesta pesquisa - assume a contribuição para o desenvolvimento de visão compartilhada e direções estratégicas, por formar redes com pessoas fundamentais, e motivando-as a promover mudanças que melhorem a saúde e reduzam iniquidades ${ }^{(5)}$. Liderar é uma atividade complexa e que requer atributos variados. Alguns profissionais têm características inerentes de líder e necessitam aperfeiçoá-las ao seu contexto; outros necessitam de maior empenho no desenvolvimento dessas habilidades ${ }^{(11)}$. Para que existam profissionais com esse domínio, desde a graduação, devem ser empregados métodos que estimulem o aluno a trabalhar em equipe, negociar, resolver conflitos, tomar decisões, buscar novos conhecimentos e dedicar-se com responsabilidade no desenvolvimento de habilidades essenciais para ser líder.

O diagnóstico foi outro domínio que esteve presente nos saberes e prática dos docentes nesta pesquisa. Ele propicia a aquisição de informações essenciais do público assistido, posto que são indispensáveis para subsidiar o planejamento e a implementação das ações. Os profissionais da saúde devem se apropriar das metodologias de pesquisa, epidemiologia e estatística, bem como dos diferentes métodos de interpretação das informações levantadas ${ }^{(12)}$.

Diagnosticar refere-se às necessidades e às potencialidades de parceria com os atores/parceiros sociais, no contexto dos determinantes políticos, econômicos, sociais, culturais, ambientais, comportamentais e biológicos, que promovem ou comprometem a saúde ${ }^{(5)}$. Assim, o desenvolvimento de pesquisas na área de PS, a identificação dos determinantes sociais e das necessidades de saúde que impactam a PS, presentes nas falas dos participantes do estudo, contemplaram esse domínio.

O domínio planejamento não pôde ser identificado nos saberes dos docentes. Este achado requer reflexões, uma vez que o planejamento é indispensável à prática pedagógica do professor, como instrumento organizador e norteador do processo de trabalho. Ao planejar, o docente delineia as ações para alcançar os objetivos propostos. Esse domínio também norteia a busca da autonomia, a tomada de decisão, as resoluções de problemas e contribui para a identificação das estratégias de PS apropriadas para o alcance das metas e objetivos fixados/acordados ${ }^{(13)}$.

Foi possível identificar o domínio implementação no momento em que foram mensuradas as estratégias de comunicação e desenvolvidos debates pelos participantes. O docente dispõe de recursos e materiais que facilitam o domínio da implementação de diferentes formas: oficinas, rodas de conversas, dinâmicas em grupo e palestras, entre outros. O uso de variadas estratégias oferece mais chances de despertar o interesse dos participantes, além de contribuir para seu protagonismo e seu empoderamento ${ }^{(14)}$.

O desenvolvimento de pesquisa e a prática baseada em evidência representaram a utilização do domínio avaliação e pesquisa do CompHp na prática dos docentes. Isto pode se justificar pelo elo, nas universidades, entre ensino e pesquisa. Muitas vezes os professores de graduação e, principalmente, de pós-graduação têm afinidade pelas pesquisas, inclusive por haver incentivo financeiro para projetos de pesquisa. Muitos cursos exigem como pré-requisito de avaliação o desenvolvimento de pesquisas.

A avaliação contribui para redefinir e melhorar as ações de PS, obtendo-se informações que ajudarão nas tomadas de decisões. Para isto, é necessário que o planejamento e a execução da avaliação considerem suas múltiplas finalidades, levando em conta o perfil final desejado de habilidades e competências. A escolha dos métodos deve se pautar no que se deseja avaliar e, também, nas finalidades da avaliação ${ }^{(15)}$.

A partir da identificação, neste estudo, dos domínios supracitados, evidencia-se que, se nos saberes dos docentes de enfermagem estão presentes os referidos domínios, elevam-se as possibilidades de os enfermeiros egressos da instituição estudada adquirirem competências asseguradas pelos domínios.

Chama atenção que alguns domínios do CompHP não foram encontrados nas respostas dos participantes. Embora todos os domínios tenham estado presentes na prática docente da instituição elencada para a pesquisa, não se pode afirmar que todos são exercidos de forma integrada pelos docentes estudados. 
Infere-se que a ausência de tais domínios fragilize a formação dos profissionais de enfermagem, o que acarreta posteriores dificuldades para: mobilizar processos de reivindicações a favor de indivíduos, comunidades e organizações, com a finalidade de melhorar a saúde (advocacia); desenvolver metas e objetivos de PS que possam ser medidos com base no diagnóstico das necessidades e potencialidades, em parceria com os atores/parceiros sociais (planejamento); implementar ações de PS efetivas, eficientes, culturalmente sensíveis e éticas, em parceria com os atores/parceiros sociais (implementação); e utilizar métodos de avaliação e pesquisa apropriados, em parceria com os atores/parceiros sociais, para determinar o alcance, o impacto e a efetividade das ações de PS (avaliação e pesquisa).

Diante da fragilidade de alguns domínios exercidos pelos docentes estudados, sobretudo o planejamento e a avaliação, os quais estiveram pouco frequentes nas respostas dos participantes, pode existir comprometimento no êxito das práticas de PS, tanto dos docentes como de seus discentes. Além disto, infere-se que a utilização fragmentada desses domínios tenha como resultado enfermeiros com dificuldades de também implementar domínios pouco explorados durante a graduação.

Assim, a construção e a contextualização de significados durante o processo de aprendizagem, integrando teoria e prática, proporcionam a reflexão e a teorização a partir da realidade prática concreta, desenvolvendo capacidades profissionais que possibilitam deslocar a atenção das disciplinas (até então presentes em muitos currículos). Em um currículo orientado por competência, o que importa não é a transmissão do conhecimento acumulado, mas, sim, a possibilidade de ação e a capacidade de recorrer ao que se sabe para realizar o que se projeta ${ }^{(16)}$.

Destaca-se a importância do corpo docente estar bem embasado e consciente do que a competência em PS representa. Isto porque é no plano pedagógico que se testemunham a organização e a legitimação da passagem de um ensino centrado em saberes disciplinares para um definido pela produção de competências em situações concretas e específicas, o qual capacita sujeitos a compreender e dominar e mobiliza recursos de diferentes naturezas (conhecimentos, habilidades e atitudes) ${ }^{(17)}$.

Portanto, os profissionais de saúde devem possuir competências para responder a necessidades e demandas de saúde da população em situações concretas, em uma perspectiva de desenvolvimento e aprimoramento ininterruptos durante a graduação e a atuação/experiência profissional. Isto requer a utilização de estratégias pedagógicas inovadoras, baseadas no aprendizado teórico-vivencial, que desperta, no discente, o interesse e o envolvimento em seu próprio aprendizado e, consequentemente, em sua formação ${ }^{(18)}$.

O estudo teve como limitações a utilização de um questionário como único método de coleta de dados e a baixa participação do público convidado para a pesquisa, tendo em vista a quantidade de pessoas que haviam sido convidadas.

\section{CONCLUSÃO}

Os nove domínios do Core Competencies Framework for Health Promotion estiveram presentes no discurso dos docentes, quando indagados sobre as práticas de promoção da saúde. No entanto, os domínios advocacia, planejamento, implementação e avaliação e pesquisa não se manifestaram nos saberes desses profissionais.

A divergência no discurso referente aos saberes e práticas dos domínios do Core Competencies Framework for Health Promotion pode ser justificada pela fragilidade do aporte teórico referente ao tema, o que pode implicar falha no processo de formação de enfermeiros, no que se refere às competências voltadas à promoção da saúde.

É importante repensar constantemente a prática docente em torno das ações de promoção da saúde com base no Core Competencies Framework for Health Promotion. Para isto, são indispensáveis a qualificação e a formação continuada dos docentes das diferentes disciplinas, para contribuir para a aquisição de conhecimentos, habilidades e atitudes necessárias para orientar e conduzir as práticas em torno da promoção da saúde, formando profissionais qualificados. 


\section{REFERÊNCIAS}

1. Valle ARMC. Competências do enfermeiro para ações preventivas na atenção domiciliar com ênfase nos riscos de infecção [Tese]. Ribeirão Preto: Escola de Enfermagem de Ribeirão Preto; 2013. Disponível em: http://www.teses.usp. br/teses/disponiveis/22/22132/tde-26092013-182224/pt-br.php.

2. Fragelli TB, Shimizu HE. Competências profissionais em Saúde Pública: conceitos, origens, abordagens e aplicações. Rev. bras. enferm. [Internet] 2012;65(4) [acesso em 18 jan 2018]. Disponível: http://dx.doi.org/10.1590/S003471672012000400017.

3. Barry MM, Battel-Kirk B, Dempsey C. The CompHP Core Competencies Framework for Health Promotion in Europe. Health Education \& Behavior. [Internet] 2012;39(6) [acesso em 18 jan 2018]. Disponível: http://dx.doi. org/10.1177/1090198112465620.

4. Pinheiro DGM, Scabar RG, Maeda ST, Fracolli LA, Pelicioni MC, Chiesa AM. Health promotion competencies: challenges of formation. Saude soc. [Internet] 2015;24(1) [acesso em 00 mês 0000]. Disponível: http://dx.doi. org/10.1590/S0104-12902015000100014.

5. Barry MM, Battel-Kirk B, Davison H, Dempsey C, Parish R, Schipperen M, et al. The CompHP Project Handbooks. [Internet] Paris: International Union for Health Promotion and Education; 2012 [acesso em: 18 jan 2018]. Disponível: http://www.fundadeps.org/recursos/documentos/450/CompHP_Project_Handbooks.pdf.

6. Silva KL, de Sena RR, Grillo MJ, Horta NC. Formação do enfermeiro: desafios para a promoção da saúde. Esc. Anna Nery Rev. [Internet] 2010;14(2) [acesso em 18 jan 2018]. Disponível: http://dx.doi.org/10.1590/S1414-81452010000200022.

7. Andrade RD, Mello DF, Silva MA, Ventura CA. Advocacia em saúde na atenção à criança: revisão da literatura. Rev. bras. enferm. [Internet] 2011;64(4) [acesso em 18 jan 2018]. Disponível: http://dx.doi.org/10.1590/S003471672011000400017.

8. Silva KL, de Araújo FL, Santos FB, Andrade AM, Basílio NC, de Sena RR. O que vem se falando por aí em competências no ensino da promoção da saúde na formação do enfermeiro? ABCS Health Sci. [Internet] 2015;40(3) [acesso em 18 jan 2018]. Disponível: http://dx.doi.org/10.7322/abcshs.v40i3.809.

9. Tusset D, Nogueira JAD, Rocha DG Rezende R. Análise das competências em promoção da saúde a partir do marco legal e dos discursos dos profissionais que implementamo Programa Saúde na Escolano Distrito Federal. Tempus, Actas de Saúde Colet. [Internet] 2015;9(1) [acesso em: 19 jan 2018]. Disponível: http://tempusactas.unb.br/index.php/tempus/ article/view/1701.

10. Almeida ME. Gestão de tecnologias, mídias e recursos na escola: o compartilhar de significados. Em Aberto. [Internet] 2008;21(79) [acesso em 18 jan 2018]. Disponível: http://emaberto.inep.gov.br/index.php/emaberto/article/ view/2306/2269.

11. Nascimento CD, Fonseca ISS, Moura SB, Servo MLS. Agir do enfermeiro no exercício da liderança em enfermagem: dificuldades da prática. Rev. enferm. UFPE on line. [Internet] 2008;2(4) [acesso em 18 jan 2018]. Disponível: https:// periodicos.ufpe.br/revistas/revistaenfermagem/article/view/5356/4575.

12. Pompeo DA, Rossi LA, Galvão CM. Revisão integrativa: etapa inicial do processo de validação de diagnóstico de enfermagem. Acta paul. enferm. [Internet] 2009;22(4) [acesso em 18 jan 2018]. Disponível: http://dx.doi.org/10.1590/ S0103-21002009000400014.

13. de Carvalho VL, de Oliveira AL, Alves IK, Silva RL, da Silva CB. Competências para promoção da saúde em formandos dos cursos da área da saúde. Rev. enferm. UFPE on line. [Internet] 2017;11(Suppl 8) [acesso em 18 jan 2018]. Disponível: https://periodicos.ufpe.br/revistas/revistaenfermagem/article/view/110193. 
14. Cardoso LS, Cezar-Vaz MR, da Costa VZ, Bonow CA, de Almeida MC. Promoção da saúde e participação comunitária em grupos locais organizados. Rev. bras. enferm. [Internet] 2013;66(6) [acesso em: 18 jan 2018]. Disponível: http:// dx.doi.org/10.1590/S0034-71672013000600018.

15. Panúncio-Pinto MP, Troncon LE. Avaliação do estudante - aspectos gerais. Medicina (Ribeirão Preto). [Internet] 2014;47(3) [acesso em 18 jan 2018]. Disponível: http://revista.fmrp.usp.br/artigos_2014.htm.

16. Chiesa AM, Fracolli LA, Veríssimo MLR, Zoboli ELCP, de Ávila LK, de Oliveira AAP. A construção de tecnologias de atenção em saúde com base na promoção da saúde. Rev. esc. enferm. USP. [Internet] 2009;43(2) [acesso em 18 jan 2018]. Disponível: http://dx.doi.org/10.1590/S0080-62342009000600036.

17. Mestriner Júnior W, Mestriner SF, Bulgarelli AF, Mishima SM. O desenvolvimento de competências em atenção básica à saúde: a experiência no projeto Huka-Katu. Ciênc. saúde coletiva. [Internet] 2011;16(Suppl 1) [acesso em: 18 jan 2018]. Disponível: http://dx.doi.org/10.1590/S1413-81232011000700022.

18. Spagnol CA, Soares AN, Silveira BV. Experiências pedagógicas vivenciadas na disciplina competências e habilidades pra gestão de pessoas na organização de saúde. RECOM. [Internet] 2012;2(3) [acesso em 18 jan 2018]. Disponível: http://www.seer.ufsj.edu.br/index.php/recom/article/view/246/359. 